\title{
A Markovian Canonical Form of Second-Order Matrix-Exponential Processes
}

\author{
L. Bodrog, ${ }^{\text {a }}$ A. Heindl, ${ }^{\mathrm{b}}$ G. Horváth, ${ }^{\mathrm{a}}$ M. Telek ${ }^{\mathrm{a}}$ \\ ${ }^{a}$ Department of Telecommunications, Budapest University of Technology and \\ Economics, \\ P.O. Box 91, H-1521 Budapest, Hungary \\ E-mail: \{bodrog,ghorvath,telek\}@hit.bme.hu \\ ${ }^{b}$ Institute of Computer Science, University of Erlangen-Nuremberg, \\ D-91058 Erlangen, Germany \\ E-mail: armin.heindl@informatik.uni-erlangen.de
}

Besides the fact that - by definition - matrix-exponential processes (MEPs) are more general than Markovian arrival processes (MAPs), only very little is known about the precise relationship of these processes in matrix notation. For the first time, this paper proves the persistent conjecture that - in two dimensions - the respective sets, $\operatorname{MAP}(2)$ and $\operatorname{MEP}(2)$, are indeed identical with respect to the stationary behavior. Furthermore, this equivalence extends to acyclic MAPs, i.e., $\operatorname{AMAP}(2)$, so that $A M A P(2) \equiv M A P(2) \equiv$ $M E P(2)$. For higher orders, these equivalences do not hold.

The second-order equivalence is established via a novel canonical form for the (correlated) processes. An explicit moment/correlation-matching procedure to construct the canonical form from the first three moments of the interarrival time distribution and the lag-1 correlation coefficient shows how these compact processes may conveniently serve as input models for arrival/service processes in applications.

Keywords: Markovian arrival process, matrix-exponential process, canonical representation, moment/correlation matching.

\section{Introduction}

Since their theoretic foundation in [23], a huge body of matrix-analytical techniques has been developed for queues and queueing networks, which can model complex systems of diverse fields of applications. Random times and stochastic processes, e.g., for arrivals and services, need to be represented in matrix notation. Two different notations have become popular: on the one hand, phase-type distributions (PHs) and Markovian arrival processes (MAPs, [21]), whose structure admits a probabilistic interpretation in terms of Markov chains; on the other hand, matrix-exponential distributions (MEs) and processes (MEPs, [20]), which gain more algebraic flexibility by abandoning this probabilistic interpretation. Past and ongoing research on these distributions and processes, where PHs and MAPs have received much more attention than their counterparts, identified a number 
of important properties (see e.g., $[17,10,11,8]$ ). However, neither the relationship between PHs and MEs nor that of MAPs and MEPs is fully understood.

$\mathrm{PH}$ and ME distributions have rational Laplace transforms of their densities [20,3]. For a specific matrix dimension $n \geq 1$, ME distributions of order $n$ contain $\mathrm{PH}$ distributions of order $n$, i.e., $M E(n) \supset P H(n)$. Generally, the true subset relation holds, except for the trivial case $n=1$ and for $n=2$. However, beyond $M E(2) \equiv P H(2)$, researchers have not yet succeeded to clearly identify the difference sets for $\mathrm{ME}$ and $\mathrm{PH}$ distributions.

The algebraic flexibility of ME distributions allowed to develop a general canonical form [25], where the parameters are given in terms of the first marginal moments. However, for arbitrary dimension, it is still an open problem to decide if this canonical form actually represents a valid probability distribution or not (due to out-of-bounds moments). In contrast, the Markovian structure of PHs makes it very easy to decide if a given candidate represents a valid distribution, but the overparameterization of PHs makes their construction more difficult and has led to the proposal of several structural simplifications, like acyclic PHs (APHs, [4]), to ease the moment fitting - however, often at the expense of the modeling power.

In summary for distributions, it remains an open question how general different subclasses of PH distributions are and which set of distributions the specific PH representations cover - especially with respect to their ME counterparts. Such questions have been answered only for two-state distributions. It is well-known that the set of two-state acyclic PHs (or $\mathrm{APH}(2)$ ) defines the same class as general $\mathrm{PH}(2)$ s or the structurally unrestricted $\mathrm{ME}(2)$ distributions.

Many of the above general observations carry forward to the related stochastic processes. By definition, MEPs of order $n$ (referred to as rational arrival processes in [2]) contain MAPs of order $n$, where corresponding sets obey a true subset relation for $n \geq 3$. In view of the situation for distributions, it has only been conjectured that the sets $\operatorname{MAP}(2)$ and $\operatorname{MEP}(2)$ are identical, but the equivalence $M A P(2) \equiv M E P(2)$ has not been proven yet. In this paper, we provide a proof for $M A P(2) \equiv M E P(2)$.

In analogy to the distributions, both MEPs and MAPs have their specific advantages and disadvantages with respect to their construction. The canonical form for ME distributions has been extended to MEPs in [22], which - besides the first marginal moments involves the initial correlation coefficients. For processes, already the case $n=2$ poses the decision problem whether these input parameters lie within respective bounds in order for the canonical form to represent a valid stochastic process. Inversely, a MAP candidate is easily identified as a valid stochastic process due to its Markovian underpinning, but we still lack both a general canonical form and a general fitting procedure for MAPs, even for order 2.

The first research results related to the inverse characterization of $\mathrm{MAP}(2) \mathrm{s}$ appeared in [13], where the region of the first three moments and the single correlation parameter is identified, which is permissible for a $\operatorname{MAP}(2)$ with hyperexponential marginals (i.e., where matrix $\boldsymbol{D}_{\mathbf{0}}$ is diagonal). These results are extended in [14] to acyclic MAP(2)s or $\operatorname{AMAP}(2)$ s, i.e., MAPs with APH marginals (where $\boldsymbol{D}_{\mathbf{0}}$ is triangular), via a different approach. That paper conjectures that $\operatorname{AMAP}(2)$ s might cover the whole class of arrival processes having two phases. In other words, the set $\operatorname{AMAP}(2)$ might be equivalent to the sets $\operatorname{MAP}(2)$ and $\operatorname{MEP}(2)$. 
In this paper, we introduce a special $\mathrm{MAP}(2)$ structure (that we call canonical form) that is even more special than the $\operatorname{AMAP}(2)$ form given in [14], since it has only four parameters (as opposed to five). We prove that this canonical form represents all $\operatorname{AMAP}(2)$, $\operatorname{MAP}(2)$ and $\operatorname{MEP}(2)$ processes with respect to stationary behavior. Only by reformulating the bounds of the involved correlation parameter (in another form already given in [14] for $\operatorname{AMAP}(2) \mathrm{s})$, we are able to prove that the class of $\operatorname{AMAP}(2) \mathrm{s}$ represented by the canonical form is equivalent to the class of $\operatorname{MEP}(2)$ s, which in turn ensures that $A M A P(2) \equiv M A P(2) \equiv M E P(2)$.

Besides this theoretical result together with a novel canonical form, this paper gives an explicit analytical fitting procedure to construct these processes from information on their marginal moments and their autocorrelation structure, e.g., the lag-1 correlation coefficient of interarrival times. Explicit correlation bounds for these classes are also provided. Even though the second order naturally limits the use of MEP(2)s and MAP(2)s in traffic/service modeling, we emphasize that excellent results could be obtained in practical data fitting with examples of such processes by various authors, e.g., in $[7,19,16]$. In some cases, MAP(2)s are also used as basic building blocks in more complex modeling and fitting approaches $[18,1]$. Also note the various applications of two-state Markov-modulated Poisson processes $(\mathrm{MMPP}(2) \mathrm{s})$ in teletraffic/internet engineering (e.g., [12,26]). Generally, compact models for arrival/service processes are very important to mitigate the state-space explosion problem (e.g., in Markov modeling) or to design more efficient algorithms (e.g., in traffic-based decomposition [9]). Due to their construction from moment and correlation parameters, $\operatorname{MAP}(2) \mathrm{s}$ or $\operatorname{MEP}(2)$ s are well suited for sensitivity analyses of systems with respect to single arrival/service characteristics.

The rest of the paper is organized as follows. Section 2 introduces the distributions, which have a second-order rational Laplace transform, along with their matrix representations. From this discussion, the known equivalences $M E(2) \equiv P H(2) \equiv A P H(2)$ will become obvious. In Section 3, we outline the different definitions of the stochastic processes $\operatorname{MEP}(2) s, \operatorname{MAP}(2) s$ and $\operatorname{AMAP}(2)$ s. Section 4 introduces the general canonical form for second-order arrival processes. Its correlation bounds are first derived in the context of restricted $\operatorname{AMAP}(2) \mathrm{s}$ and then shown in Section 5 to extend to $\mathrm{MEP}(2) \mathrm{s}$. This proves the equivalence $M E P(2) \equiv(A) M A P(2)$. Section 6 outlines how the theoretical results of this paper can be applied in practice and provides a fitting procedure for the general canonical form. Finally, Section 7 summarizes and concludes this paper.

\section{Distributions with second-order rational Laplace transforms}

Let $X$ be a continuous non-negative random variable with cumulative distribution function

$F(t)=\operatorname{Pr}(X<t)=1-\boldsymbol{v} e^{\boldsymbol{H}_{t}} \mathbb{I}$,

where row vector $\boldsymbol{v}$ is referred to as the initial vector, square matrix $\boldsymbol{H}$ as the generator and $\mathbb{I}$ as the closing vector. Without loss of generality (see [20]), throughout this paper we assume that the closing vector, $\mathbb{I}$, is a column vector of ones, i.e., $\mathbb{I}=[1,1, \ldots, 1]^{T}$. As $X$ is a continuous random variable, it has no probability mass at zero, i.e., $\boldsymbol{v} \mathbb{I}=1$. The density, its Laplace transform and the moments of $X$ can be computed as 
$f(t)=\boldsymbol{v} e^{\boldsymbol{H}_{t}}(-\boldsymbol{H}) \mathbb{I}$

$f^{*}(s)=E\left(e^{-s X}\right)=\boldsymbol{v}(s \boldsymbol{I}-\boldsymbol{H})^{-1}(-\boldsymbol{H}) \mathbb{I}$,

$\mu_{n}=E\left(X^{n}\right)=n ! \boldsymbol{v}(-\boldsymbol{H})^{-n} \mathbb{I}$.

In this paper, the cardinality of vector $\boldsymbol{v}$ and of matrix $\boldsymbol{H}$ is assumed to be 2. We consider the following three cases:

- If $f(t) \geq 0$ and $\int_{0}^{\infty} f(t) d t=1$, then $X$ has a $\mathrm{ME}(2)$ distribution. The elements of $\boldsymbol{v}$ and $\boldsymbol{H}$ may be arbitrary real numbers.

- If $\boldsymbol{v}=\boldsymbol{\pi}$ is a probability vector and $\boldsymbol{H}=\boldsymbol{A}$ is a transient Markovian generator matrix (i.e., the generator matrix of a transient continuous-time Markov chain (CTMC)), then $X$ has a $\mathrm{PH}(2)$ distribution.

- If $\boldsymbol{v}=\boldsymbol{\pi}$ is a probability vector and $\boldsymbol{H}=\boldsymbol{A}$ is an acyclic transient Markovian generator matrix, then $X$ has an $\operatorname{APH}(2)$ distribution.

By $\operatorname{ME}(2), \operatorname{PH}(2)$ and $\operatorname{APH}(2)$, we also denote the three corresponding sets of distributions. Generally, we use different notations for the matrix-exponential (ME, MEP) and the Markovian (PH, MAP) representations to emphasize that different constraints apply to them.

Recall that $\boldsymbol{\pi}$ is a probability vector when $\boldsymbol{\pi}_{i} \geq 0, \boldsymbol{\pi} \mathbb{I}=1$ (where the latter condition is fulfilled a priori). Matrix $\boldsymbol{A}$ is a transient Markovian generator when $\boldsymbol{A}_{i i}<0, \boldsymbol{A}_{i j} \geq 0$ for $i \neq j, \boldsymbol{A} \mathbb{I} \leq \mathbf{0}, \boldsymbol{A} \mathbb{I} \neq \mathbf{0}$. Matrix $\boldsymbol{A}$ is an acyclic transient Markovian generator, when $\boldsymbol{A}$ is a transient Markovian generator matrix and there is no loop in $\boldsymbol{A}$, i.e., $\boldsymbol{A}_{12}$ or $\boldsymbol{A}_{21}$ is zero. Without loss of generality, we consider upper triangular acyclic generators in this paper, i.e., $\boldsymbol{A}_{21}=0$. Scalars like $\boldsymbol{A}_{i j}$ denote the $i j$ th element of matrix $\boldsymbol{A}$.

To ensure that $f(t)$ in (1) is a density function, $\boldsymbol{H}$ generally has to fulfill the necessary condition that its eigenvalues are real and negative (consequently $\boldsymbol{H}$ is non-singular). In the second-order case, we can fully classify these density functions:

Theorem 1 [6] For second-order representations (1), the Laplace transform $f^{*}(s)$ has the form

$f^{*}(s)=\frac{1+s / \sigma}{\left(1+s / \lambda_{1}\right) \cdot\left(1+s / \lambda_{2}\right)}$.

Function $f(t)$ in (1) represents a density function, if and only if $\lambda_{1}, \lambda_{2}$ and $\sigma$ are all real and

$0<\min \left(\lambda_{1}, \lambda_{2}\right) \leq \sigma \leq \infty$

The poles and the zero of $f^{*}(s)$ are $-\lambda_{1},-\lambda_{2}$ and $-\sigma$, respectively, with all of them being on the negative real axis. Without loss of generality, let $\lambda_{1} \leq \lambda_{2}$ (or $-\lambda_{1} \geq-\lambda_{2}$ ). The density function can be written as

$f(t)= \begin{cases}\frac{\sigma-\lambda_{1}}{\lambda_{2}-\lambda_{1}} \cdot \frac{\lambda_{2}}{\sigma} \cdot \lambda_{1} e^{-\lambda_{1} t}+\frac{\sigma-\lambda_{2}}{\lambda_{1}-\lambda_{2}} \cdot \frac{\lambda_{1}}{\sigma} \cdot \lambda_{2} e^{-\lambda_{2} t}, & \text { if } \lambda_{1}<\lambda_{2}, \\ \frac{\lambda_{1}}{\sigma} \lambda_{1} e^{-\lambda_{1} t}+\left(1-\frac{\lambda_{1}}{\sigma}\right) \lambda_{1}^{2} t e^{-\lambda_{1} t}, & \text { if } \lambda_{1}=\lambda_{2} .\end{cases}$ 


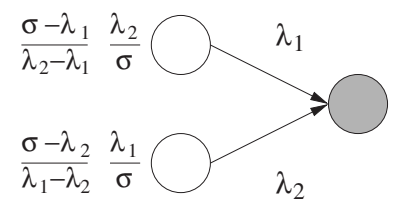

Figure 1. ME(2) representation (7)

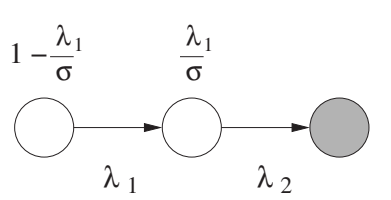

Figure 2. $\mathrm{ME}(2)$ representation (8)

A matrix representation of distributions with the rational Laplace transform (4) does not necessarily have a probabilistic/Markovian structure for vector $\boldsymbol{v}$ and matrix $\boldsymbol{H}$. This can already be seen from the visualization of the two-branch canonical representation

$\boldsymbol{v}=\left[\begin{array}{cc}\frac{\sigma-\lambda_{1}}{\lambda_{2}-\lambda_{1}} \cdot \frac{\lambda_{2}}{\sigma} & \frac{\sigma-\lambda_{2}}{\lambda_{1}-\lambda_{2}} \cdot \frac{\lambda_{1}}{\sigma}\end{array}\right], \boldsymbol{H}=\left[\begin{array}{cc}-\lambda_{1} & 0 \\ 0 & -\lambda_{2}\end{array}\right]$

in Figure 1. This representation can be interpreted as a transient CTMC (more precisely, a Bernoulli mixture of two exponentials), if and only if $\lambda_{1} \leq \sigma \leq \lambda_{2}$, where $\lambda_{1} \neq \lambda_{2}$ in order to avoid stochastic equivalence with the scalar exponential distribution. Only in this case, which corresponds to a squared coefficient of variation $c_{v}^{2}=\frac{\mu_{2}}{\mu_{1}^{2}}-1 \geq 1, \boldsymbol{v}$ is probabilistic. In the other permissible range according to (5), $\sigma>\lambda_{2}$, however, (7) is still a valid $\mathrm{ME}(2)$ representation for a density function, but $\boldsymbol{v}$ is not a probability vector.

The notational differences between phase-type and matrix-exponential representations could be highlighted by many other representations, where e.g., both the row sum and the diagonal element of $\boldsymbol{H}$ may be positive in contrast with phase-type generators. Despite these differences, the three classes for second-order distributions, i.e., acyclic $\mathrm{PH}(2)$, arbitrary $\mathrm{PH}(2)$ and $\mathrm{ME}(2)$, can easily be shown to be identical, which we formally state in the following theorem.

Theorem 2 The distribution sets ME(2), PH(2) and APH(2) are equivalent, i.e., $M E(2) \equiv P H(2) \equiv A P H(2)$.

Proof: $\quad$ Based on the definition of these classes, we have $M E(2) \supset P H(2) \supset A P H(2)$. Here, we only prove that any $\mathrm{ME}(2)$ distribution has an $\mathrm{APH}(2)$ representation.

Let us rewrite the Laplace transform $f^{*}(s)$ of the density function (see (4)) as

$$
f^{*}(s)=\frac{1-\lambda_{1} / \sigma}{\left(1+s / \lambda_{1}\right) \cdot\left(1+s / \lambda_{2}\right)}+\frac{\lambda_{1} / \sigma}{\left(1+s / \lambda_{2}\right)} .
$$

This structure reveals an analogy to a Laplace transform of a Bernoulli mixture of a hypoexponential density and an exponential density, which leads us to the following matrixexponential representation

$\boldsymbol{v}=\left[\begin{array}{ll}p & 1-p\end{array}\right](=\boldsymbol{\pi}), \boldsymbol{H}=\left[\begin{array}{cc}-\lambda_{1} & \lambda_{1} \\ 0 & -\lambda_{2}\end{array}\right] \quad(=\boldsymbol{A})$,

with $p=1-\frac{\lambda_{1}}{\sigma}$. Figure 2 visualizes this acyclic $M E(2)$ representation. It is easily verified that (1) with these settings for $\boldsymbol{v}$ and $\boldsymbol{H}$ yields (6). Due to condition (5), i.e., $\lambda_{1} \leq \sigma$, 
it follows $0 \leq \frac{\lambda_{1}}{\sigma} \leq 1$ so that representation (8) is indeed a valid $\operatorname{APH}(2)$ representation $(\boldsymbol{\pi}, \boldsymbol{A})$.

Thus, we can represent any $\mathrm{ME}(2)$ distribution as an $\mathrm{APH}(2)$ distribution via (8) based on which we identify a $\operatorname{ME}(2)$ distribution with the triple $\left\{p, \alpha, \lambda_{1}\right\}$, where $\alpha=\lambda_{1} / \lambda_{2}$. The valid ranges of the parameters are $0 \leq p \leq 1,0 \leq \alpha \leq 1, \lambda_{1}>0$. In this triple, $p$ and $\alpha$ define the "shape" of the distribution and $\lambda_{1}$ affects only its "intensity". Introducing parameter $\alpha$ will also help us to formulate bounds and fitting procedures more simply in the sequel of this paper.

The $(\boldsymbol{\pi}, \boldsymbol{A})$-representation is not unique. A set of different APH representations can describe the same distribution. For example, for later use introduce

$\boldsymbol{\pi}(a)=\left[\frac{p}{1-\alpha a} \quad 1-\frac{p}{1-\alpha a}\right], \quad \boldsymbol{A}(a)=\left[\begin{array}{cc}-\lambda_{1} & (1-a) \lambda_{1} \\ 0 & -\lambda_{2}\end{array}\right]$

which results in a valid APH representation when $0 \leq a \leq \min \{1,(1-p) / \alpha\}$. If $a=1$, we require $\alpha \neq 1$. Otherwise, the two-dimensional representation (9) reduces to a (scalar) exponential distribution.

\section{Arrival processes of second order}

Let $X(t)$ be the number of arrivals at time $t$ in an interval-stationary arrival process, defined by matrices $\boldsymbol{H}_{\mathbf{0}}$ and $\boldsymbol{H}_{\mathbf{1}}$, whose sequence of interarrival times is $X_{0}, X_{1}, \ldots$ The joint density of $X_{0}, X_{1}, \ldots, X_{k}$ is

$f\left(x_{0}, x_{1}, \ldots, x_{k}\right)=\boldsymbol{v} e^{\boldsymbol{H}_{\mathbf{0}} x_{0}} \boldsymbol{H}_{\mathbf{1}} e^{\boldsymbol{H}_{\mathbf{0}} x_{1}} \boldsymbol{H}_{\mathbf{1}} \ldots e^{\boldsymbol{H}_{\mathbf{0}} x_{k}} \boldsymbol{H}_{\mathbf{1}} \mathbb{I}$,

where $\boldsymbol{v}$ is the solution of $\boldsymbol{v}\left(-\boldsymbol{H}_{\mathbf{0}}\right)^{-1} \boldsymbol{H}_{\mathbf{1}}=\boldsymbol{v}$ and $\boldsymbol{v} \mathbb{I}=1$.

Again, we focus on the cardinality of 2 for $\boldsymbol{H}_{\mathbf{0}}$ and $\boldsymbol{H}_{\mathbf{1}}$. Similar to the previous section, we consider three cases:

- If $f\left(x_{0}, x_{1}, \ldots, x_{k}\right) \geq 0 \quad \forall k \geq 0$ and $\forall x_{1}, x_{2}, \ldots, x_{k} \geq 0$ and $\int_{x_{1}} \ldots \int_{x_{k}} f\left(x_{0}, x_{1}, \ldots, x_{k}\right) d x_{1} \ldots d x_{k}=1 \quad \forall k \geq 0$, then $X(t)$ is a matrixexponential process, $\operatorname{MEP}(2)$.

- If $\boldsymbol{H}_{\mathbf{0}}=\boldsymbol{D}_{\mathbf{0}}$ is a transient Markovian generator matrix and $\boldsymbol{H}_{\mathbf{1}}=\boldsymbol{D}_{\mathbf{1}} \geq 0$, such that $-\boldsymbol{D}_{\mathbf{0}} \mathbb{I}=\boldsymbol{D}_{\mathbf{1}} \mathbb{I}$, then $X(t)$ is a Markovian arrival process, $\operatorname{MAP}(2)$.

- If $\boldsymbol{H}_{\mathbf{0}}=\boldsymbol{D}_{\mathbf{0}}$ is an acyclic transient Markovian generator matrix and $\boldsymbol{H}_{\mathbf{1}}=\boldsymbol{D}_{\mathbf{1}} \geq$ 0 , such that $-\boldsymbol{D}_{\mathbf{0}} \mathbb{I}=\boldsymbol{D}_{\mathbf{1}} \mathbb{I}$, then $X(t)$ is an acyclic Markovian arrival process, $\operatorname{AMAP}(2)$.

In analogy to the distribution sets $\mathrm{ME}(2), \mathrm{PH}(2)$ and $\mathrm{APH}(2)$, we also denote the above three sets of processes by $\operatorname{MEP}(2), \operatorname{MAP}(2)$ and $\operatorname{AMAP}(2)$, respectively. Whether these acronyms are used for the specific set or an element thereof will be apparent from the context.

When $X(t)$ is a $\operatorname{MEP}(2)$, it has the following properties: 
- The stationary interarrival time distribution is matrix-exponential with parameters $\boldsymbol{v}$ and $\boldsymbol{H}_{\mathbf{0}}$. Therefore, $\boldsymbol{H}_{\mathbf{0}}$ fulfills the conditions of ME distributions provided in the previous section.

- Starting from an arbitrary initial vector $\left(\boldsymbol{v}_{\mathbf{0}}\right)$, the respective initial vectors at the consecutive interarrivals $\left(\boldsymbol{v}_{\mathbf{1}}, \boldsymbol{v}_{\mathbf{2}}, \ldots\right)$ satisfy $\boldsymbol{v}_{\boldsymbol{i}}=\boldsymbol{v}_{\boldsymbol{i}-\mathbf{1}} \boldsymbol{G}$, where $\boldsymbol{G}=\left(-\boldsymbol{H}_{\mathbf{0}}\right)^{-1} \boldsymbol{H}_{\mathbf{1}}$. Matrix $\boldsymbol{G}$ has the following properties: $\boldsymbol{v} \boldsymbol{G}=\boldsymbol{v}$ and $\boldsymbol{G} \mathbb{I}=\mathbb{1}$.

- $\mathbb{I}=G \mathbb{I}$ implies that the respective initial vectors of the consecutive arrivals $\left(\boldsymbol{v}_{\mathbf{1}}, \boldsymbol{v}_{\mathbf{2}}, \ldots\right)$ satisfy $\boldsymbol{v}_{\boldsymbol{i}} \mathbb{I}=1$, if $\boldsymbol{v}_{\mathbf{0}} \mathbb{I}=1$.

$-\mathbb{I}=\boldsymbol{G} \mathbb{I}=\left(-\boldsymbol{H}_{\mathbf{0}}\right)^{-1} \boldsymbol{H}_{\mathbf{1}} \mathbb{I}$ implies $-\boldsymbol{H}_{\mathbf{0}} \mathbb{I}=\boldsymbol{H}_{\mathbf{1}} \mathbb{I}$.

When $X(t)$ is a $\operatorname{MAP}(2)$, it has the following additional properties:

- The phases of the system at arrival epochs form a DTMC with transition probability matrix $\boldsymbol{P}=\left(-\boldsymbol{D}_{\mathbf{0}}\right)^{-1} \boldsymbol{D}_{\mathbf{1}}$, i.e., the elements of $\boldsymbol{P}$ are between 0 and $1(\boldsymbol{P}$ is a stochastic matrix).

- $\boldsymbol{v}=\boldsymbol{\pi}$ is a probability vector. It is the stationary distribution of the embedded DTMC, i.e., $\boldsymbol{\pi} \boldsymbol{P}=\boldsymbol{\pi}, \boldsymbol{\pi} \mathbb{I}=1$.

The major differences of the MEP case and the MAP case are the following. The row sum and the diagonal element of $\boldsymbol{H}_{\mathbf{0}}$ can be positive, the elements of $\boldsymbol{v}$ and $\boldsymbol{G}$ can be negative or greater than one and $\boldsymbol{H}_{\mathbf{1}}$ can contain negative elements. Note, however, that row sums of $\boldsymbol{H}_{\mathbf{0}}+\boldsymbol{H}_{\mathbf{1}}$ must be zero in both cases.

Since the interarrival times of a $\operatorname{MEP}(2)$ have a $\operatorname{ME}(2)$ distribution with generator $\boldsymbol{H}_{\mathbf{0}}$ and initial vector $\boldsymbol{v}$, the moments of the interarrival times are (in accordance with (3)):

$\mu_{n}=n ! \boldsymbol{v}\left(-\boldsymbol{H}_{\mathbf{0}}\right)^{-n} \mathbb{I}$.

If $X(t)$ is a $\operatorname{MEP}(2)(\operatorname{MAP}(2))$, matrix $\boldsymbol{G}(\boldsymbol{P})$ has two eigenvalues: 1 and $\gamma$. Parameter $\gamma$ defines the geometric decay of the lag-k correlation function [15]:

$\operatorname{Corr}\left(X_{0}, X_{k}\right)=\frac{E\left[\left(X_{0}-E[X]\right)\left(X_{k}-E[X]\right)\right]}{\operatorname{Var}[X]}=\gamma^{k} \cdot \frac{\frac{\mu_{2}}{2}-\mu_{1}^{2}}{\mu_{2}-\mu_{1}^{2}}$,

where random variable $X$ stands for a generic interarrival time. Since autocorrelation functions are necessarily non-divergent, eigenvalue $\gamma$ is limited to $-1 \leq \gamma<1$ a priori.

The consecutive discussions are based on the observation that the first three moments of the interarrival time (or equivalently $\left.\lambda_{1}, \alpha, p\right)$ and the lag- 1 correlation coefficient (or equivalently $\gamma$, according to (12)) uniquely define the stationary behavior of $\operatorname{MEP}(2) \mathrm{s}[5]$.

\section{Canonical AMAP(2)s}

In Section 4.1, we present the general canonical form for second-order processes. This canonical form at first sight appears to be even more constrained than arbitrary $\operatorname{AMAP}(2)$ s, due to an enforced zero element in matrix $\boldsymbol{D}_{\mathbf{1}}$ besides the upper triangular matrix $\boldsymbol{D}_{\mathbf{0}}$. But we will show later in Section 5 that every $\operatorname{MEP}(2)$ can be transformed 
to this canonical form. This proves the equivalence of classes $\operatorname{AMAP}(2), \operatorname{MAP}(2)$ and $\operatorname{MEP}(2)$. Until this proof is completed, we refer to all processes being represented by the canonical form as canonical $\operatorname{AMAP}(2) \mathrm{s}$.

In Section 4.2, we derive the correlation bounds for canonical $\operatorname{AMAP}(2) \mathrm{s}$. We achieve this by basing the original representation on a parameter set which involves the correlation parameter $\gamma$. This new parameterization enables us to formulate the correlation bounds in a simple form. In fact, it is this simplicity of the bounds that allows us to show their validity for $\operatorname{MEP}(2) \mathrm{s}$ in Section 5 .

\subsection{The canonical form of second-order processes}

We first define the general canonical form. The representation is based on the rate parameters $\lambda_{1}$ and $\lambda_{2}$ and probabilities $a$ and $b$, where $a$ corresponds to the parameter with the same name in the APH representation (9). Dependent on the characteristics of the correlation structure (see Corollary 1 below), there are two variants of the canonical form.

Definition 1 The first canonical representation of $M A P(2) s$ is defined as follows:

$\boldsymbol{D}_{\mathbf{0}}=\left[\begin{array}{cc}-\lambda_{1} & (1-a) \lambda_{1} \\ 0 & -\lambda_{2}\end{array}\right], \quad \boldsymbol{D}_{\mathbf{1}}=\left[\begin{array}{cc}a \lambda_{1} & 0 \\ (1-b) \lambda_{2} & b \lambda_{2}\end{array}\right]$.

The second canonical form is given by

$\boldsymbol{D}_{\mathbf{0}}=\left[\begin{array}{cc}-\lambda_{1} & (1-a) \lambda_{1} \\ 0 & -\lambda_{2}\end{array}\right], \quad \boldsymbol{D}_{\mathbf{1}}=\left[\begin{array}{cc}0 & a \lambda_{1} \\ b \lambda_{2} & (1-b) \lambda_{2}\end{array}\right]$

where $0<\lambda_{1} \leq \lambda_{2}, 0 \leq a \leq 1$ and $0 \leq b \leq 1$. Additionally, we require that

- $a, b \neq 1$ in the first canonical form (for recurrency) and

- $b \neq 0$ in the second canonical form (for recurrency) and

- $\lambda_{1} \neq \lambda_{2}$, if $a=1$ in the second canonical form.

For correlated processes, $a$ and $b$ must be nonzero.

Several characteristics of the canonical form depend only on parameters $a$ and $b$ :

Corollary 1 The correlation parameter $\gamma$ of the first canonical form is given by

$\gamma=a b$.

The correlation parameter $\gamma$ of the second canonical form is given by

$\gamma=-a b$.

The phase probability vector at stationary arrival epochs in case of the first canonical form is

$\boldsymbol{\pi}=\left[\begin{array}{ll}\frac{1-b}{1-a b} & \frac{b-a b}{1-a b}\end{array}\right]$,

In case of the second canonical form, it is

$\boldsymbol{\pi}=\left[\frac{b}{1+a b} \quad 1-\frac{b}{1+a b}\right]$. 


\section{Basing the representations on parameters $\lambda_{1}, \alpha, p, \gamma$}

We may also express the canonical forms in terms of the four parameters $\lambda_{1}, \alpha, p, \gamma$. Parameters $\alpha=\frac{\lambda_{1}}{\lambda_{2}}$ and $p$ were already introduced in Section 2 (see equation (9)). To complete the transformation, we still need to express parameters $a$ and $b$ in terms of $\lambda_{1}, \alpha, p, \gamma$.

According to (9), we can get several representations of the same $\operatorname{APH}(2)$ distribution with different settings of $a$ in its valid range. On the other hand, the phase probability vector $\boldsymbol{\pi}$ of the canonical $\operatorname{AMAP}(2)$ is given by (15) for positive $\gamma$ and (16) for negative $\gamma$. (For $\gamma=0$, the canonical $\operatorname{AMAP}(2)$ simply reduces to an $\operatorname{APH}(2)$ renewal process with trivial relations, e.g., $a=b=0$ in the first canonical form.) By equating the initial vector $\boldsymbol{\pi}(a)$ of the marginal $\mathrm{APH}(2)$ distribution and the phase probability vector $\boldsymbol{\pi}$ of the canonical $\operatorname{AMAP}(2)$, we may determine parameters $a$ and $b$ in terms of $\alpha, p, \gamma$.

We have to distinguish two cases dependent on the sign of $\gamma$ :

Case $\gamma>0$ :

From $\boldsymbol{\pi}(a)=\boldsymbol{\pi}$ (see equation (15)), we get

$\frac{p}{1-\alpha a}=\frac{1-b}{1-a b}$.

The second equation is identical to (13): $\gamma=a b$.

This set of equations has two solutions, where $a, b>0$ holds in both solutions. If there is a valid solution at all (i.e., $\gamma$ is permissible), then it is the following one:

$\begin{aligned} a & =\frac{1}{2 \alpha}\left(1+\alpha \gamma-p(1-\gamma)-\sqrt{(1+\alpha \gamma-p(1-\gamma))^{2}-4 \alpha \gamma}\right), \\ b & =\frac{1}{2}\left(1+\alpha \gamma-p(1-\gamma)+\sqrt{(1+\alpha \gamma-p(1-\gamma))^{2}-4 \alpha \gamma}\right) .\end{aligned}$

Case $\gamma<0$ :

From $\boldsymbol{\pi}(a)=\boldsymbol{\pi}$ (see equation (16)), we get

$\frac{p}{1-\alpha a}=\frac{b}{1+a b}$.

The second equation is identical to (14): $\gamma=-a b$.

For permissible $\gamma$, there is only one solution in this case:

$a=\frac{-\gamma}{p(1-\gamma)-\alpha \gamma}$

$b=p(1-\gamma)-\alpha \gamma$.

Thus, both canonical forms in Definition 1 can also be expressed in terms of $\lambda_{1}, \alpha, p, \gamma$ as opposed to $\lambda_{1}, \lambda_{2}, a, b$. Since $\lambda_{1}, \lambda_{2}$ are positive rates and $a, b$ must be probabilities, the Markovian nature of the original canonical forms can be decided easily a priori based on the values of these parameters. The situation is different for the representations based on 
$\lambda_{1}, \alpha, p, \gamma$. Whereas by definition it must hold that $\lambda_{1}>0$ and $\alpha, p \in[0,1]$, the permissible range of $\gamma$ is not obvious.

In the next subsection, we exploit the knowledge about the other parameters to determine which maximal and minimal values $\gamma$ may assume so that the canonical $\operatorname{AMAP}(2)$ representations based on $\lambda_{1}, \alpha, p, \gamma$ are valid Markovian arrival processes.

\subsection{Correlation bounds of the canonical $\operatorname{AMAP}(2) \mathrm{s}$}

In the following, we derive upper and lower bounds for the correlation parameter $\gamma$ in terms of the shape parameters $\alpha$ and $p$. It turns out that these bounds are independent of the rate parameter $\lambda_{1}$.

During the derivation of both upper and lower $\gamma$-bounds, we follow the same idea. Essentially, we translate the constraints on parameters $a$ and $b$, i.e., $0 \leq a, b \leq 1$ (with the specific exceptions mentioned in Definition 1) to constraints on parameter $\gamma$. As the main step to this end, we express $\gamma$ as a function of parameter $a$, and compute the (permissible) values of $a$ (in terms of $\alpha$ and $p$ ) that produce the maximal and the minimal value of $\gamma$.

As we will see, specific choices of $\alpha$ and $p$ restrict the permissible range of probability parameter $a$ for $c_{v}^{2}<1$. Therefore, we investigate these constraints of parameter $a$ first.

With $\alpha$ and $p$ given, the condition that $\boldsymbol{\pi}(a)$ in (9) has to be a probability vector limits the range of $a$ in $[0,1]$. For the first vector component, $\frac{p}{1-\alpha a} \geq 0$ always holds, if $0 \leq a \leq 1$. From $\frac{p}{1-\alpha a} \leq 1$, we obtain $a \leq \min \{1,(1-p) / \alpha\}$. The next lemma gives an easy-to-check condition to identify the upper limit of $a$ :

Lemma $1 c_{v}^{2}<1$, if and only if $\alpha>1-p$.

Proof: The squared coefficient of variation $c_{v}^{2}$ can be expressed in terms of the parameters $\alpha$ and $p$ as follows:

$1-c_{v}^{2}=2-\frac{\mu_{2}}{\mu_{1}^{2}}=\frac{4 p \lambda_{2}}{\left(\lambda_{1}+p \lambda_{2}\right)^{2}}\left(\lambda_{1}-(1-p) \lambda_{2}\right)=\frac{4 p}{(\alpha+p)^{2}}(\alpha-(1-p))$.

Here, moments $\mu_{1}$ and $\mu_{2}$ are computed from (3) with $\mathrm{APH}(2)$ representation (9). Observing that the fraction on the right-hand side (rhs) is always positive proves the lemma.

Thus, with Lemma 1, the valid ranges of $a$ are

$\begin{array}{ll}a \in\left[0, \frac{1-p}{\alpha}\right], & \text { if } c_{v}^{2}<1, \\ a \in[0,1], & \text { if } c_{v}^{2} \geq 1 .\end{array}$

In the derivations of $\gamma$-bounds, we at least have to distinguish the two cases $c_{v}^{2}<1$ and $c_{v}^{2}>1$ for both lower and upper $\gamma$-bounds. The derivations in Appendix A reveal that for $c_{v}^{2}>1$ (hyperexponential marginal) and $\gamma<0$ (lower bound) we additionally have to treat the subcases $\frac{1-p}{p+\alpha}<1$ and $\frac{1-p}{p+\alpha} \geq 1$.

Correspondingly, Figure 3 depicts the partitioning of the $(p, \alpha)$-plane into three areas I, II, III, for which lower and upper $\gamma$-bounds are derived separately (see Appendix A). Table 1 summarizes these results of Appendix A. 


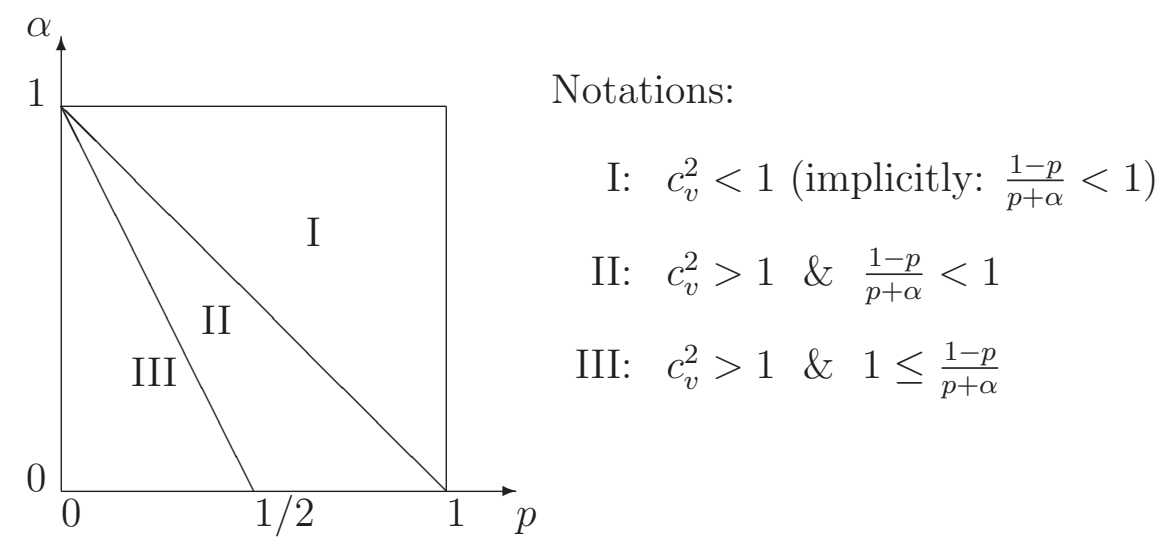

Figure 3. Fragmentation of the $(p, \alpha)$-plane in the unit square

Table 1

Lower and upper $\gamma$-bounds for the three areas in the $(p, \alpha)$-plane

\begin{tabular}{llll}
\hline area & conditions & lower $\gamma$-bound & upper $\gamma$-bound \\
\hline I & $c_{v}^{2}<1$ & $-\frac{1-p}{p+\alpha}$ & $\frac{\alpha+p(\alpha+p-1)-2 \sqrt{p \alpha(-1+p+\alpha)}}{(p+\alpha)^{2}}$ \\
II & $c_{v}^{2}>1 \& \frac{1-p}{p+\alpha}<1$ & $-\frac{1-p}{p+\alpha}$ & 1 \\
III & $c_{v}^{2}>1 \& 1 \leq \frac{1-p}{p+\alpha}$ & $\frac{p}{p+\alpha-1}$ & 1 \\
\hline
\end{tabular}

Finally, we point out that the correlation bounds of the set $\operatorname{AMAP}(2)$ were given in [14] in terms of the first three moments of the marginal $\mathrm{APH}(2)$ distribution. Indeed, these bounds can be transformed to the ones for canonical $\operatorname{AMAP}(2)$ s given in Table 1. The more compact structure of the canonical form in Definition 1 (with one parameter less than in [14]) significantly simplifies the derivation of $\gamma$-bounds. In fact, derivations were only sketched in [14], while we are able to give explicit proofs in this paper. More importantly, it is only the new parameterization (in terms of $\alpha$ and $p$ ) of these bounds that enables us to show the identity relationship between $\operatorname{MEP}(2) \mathrm{s}$ and $\operatorname{AMAP}(2) \mathrm{s}$ in Section 5 .

\section{Equivalence of $\operatorname{MEP}(2)$ and $\operatorname{AMAP}(2)$}

In this section, we prove the identity of the sets $\operatorname{MEP}(2)$ and canonical $\operatorname{AMAP}(2)$. From this fact, the central result of this paper actually follows, namely the equivalences $M E P(2) \equiv M A P(2) \equiv A M A P(2)$. We achieve this by deriving necessary constraints, which apply to the correlation parameter of an arbitrary $\operatorname{MEP}(2)$. The fact that the $\operatorname{MEP}(2)$ correlation range does not exceed that of canonical $\operatorname{AMAP}(2) \mathrm{s}$ implies that $\operatorname{MEP}(2)$ is also a subset of canonical $A M A P(2)$. As $M E P(2) \supset M A P(2) \supset A M A P(2) \supset$ canonical $A M A P(2)$ holds by definition, the identities are then proven.

Similar to $\mathrm{ME}(2)$ distributions, the $\left(\boldsymbol{H}_{\mathbf{0}}, \boldsymbol{H}_{\mathbf{1}}\right)$-representation of $\mathrm{MEP}(2)$ processes is not unique. In order to evaluate some necessary constraints of the $\operatorname{MEP}(2)$ class, we 
need to start from a non-degenerate representation of the interarrival time distribution. A representation is degenerate, if $\boldsymbol{v}_{i}=0$ or $\left\{\boldsymbol{H}_{\mathbf{0}} \mathbb{I}\right\}_{i}=0$ for $i=1$ or $i=2$. The acyclic representation (8) is degenerate, while the one in (9) is non-degenerate for all $0<a<(1-p) / \alpha$. We start from the following non-degenerate representation $\boldsymbol{H}_{\mathbf{0}}, \boldsymbol{H}_{\mathbf{1}}$, whose interarrival time distribution is according to (9):

$$
\boldsymbol{v}=\left[\frac{p \lambda_{2}}{\lambda_{2}-\lambda_{1} a}, 1-\frac{p \lambda_{2}}{\lambda_{2}-\lambda_{1} a}\right], \boldsymbol{H}_{\mathbf{0}}=\left[\begin{array}{cc}
-\lambda_{1}(1-a) \lambda_{1} \\
0 & -\lambda_{2}
\end{array}\right], \boldsymbol{H}_{\mathbf{1}}=\left[\begin{array}{cc}
a \lambda_{1}\left(1-q_{1}\right) & a \lambda_{1} q_{1} \\
\lambda_{2} q_{2} & \lambda_{2}\left(1-q_{2}\right)
\end{array}\right] .
$$

From any different $\operatorname{MEP}(2)$ representation, $\left(\hat{\boldsymbol{H}}_{\mathbf{0}}, \hat{\boldsymbol{H}}_{\mathbf{1}}\right)$, this representation can be obtained through a similarity transform with a matrix $\boldsymbol{B}$, i.e., $\boldsymbol{H}_{\mathbf{0}}=\boldsymbol{B}^{-1} \hat{\boldsymbol{H}}_{\mathbf{0}} \boldsymbol{B}, \boldsymbol{H}_{\mathbf{1}}=\boldsymbol{B}^{-1} \hat{\boldsymbol{H}}_{\mathbf{1}} \boldsymbol{B}$, where matrix $\boldsymbol{B}$ satisfies $\boldsymbol{B} \mathbb{I}=\mathbb{I}$ and $\left\{\boldsymbol{B}^{-1} \hat{\boldsymbol{H}}_{\mathbf{0}} \boldsymbol{B}\right\}_{21}=0$. If $\left(\hat{\boldsymbol{H}}_{\mathbf{0}}, \hat{\boldsymbol{H}}_{\mathbf{1}}\right)$ and $\left(\boldsymbol{H}_{\mathbf{0}}, \boldsymbol{H}_{\mathbf{1}}\right)$ represent the same process, such a matrix $\boldsymbol{B}$ exists.

Note that $-\boldsymbol{H}_{\mathbf{0}} \mathbb{I}=\boldsymbol{H}_{\mathbf{1}} \mathbb{I}$, as required (see Section 3). The eigenvalues of $\left(-\boldsymbol{H}_{\mathbf{0}}\right)^{-1} \boldsymbol{H}_{\mathbf{1}}$ are 1 and $a\left(1-q_{1}-q_{2}\right)$. Recall from Section 3 that the eigenvalue of $\left(-\boldsymbol{H}_{\mathbf{0}}\right)^{-1} \boldsymbol{H}_{\mathbf{1}}$ less than 1 corresponds to $\gamma$. From $\gamma=a\left(1-q_{1}-q_{2}\right)$ and $\boldsymbol{v}\left(-\boldsymbol{H}_{\mathbf{0}}\right)^{-1} \boldsymbol{H}_{\mathbf{1}}=\boldsymbol{v}$, we have

$q_{2}=\frac{p(\gamma-1)}{a \alpha-1}$ and $q_{1}=1-\frac{\gamma}{a}-\frac{p(\gamma-1)}{a \alpha-1}$.

At this point, with the $\mathrm{ME}(2)$ distribution being fixed, the correlation parameter $\gamma$ is the only "free" parameter in representation (21). The main constraint that limits $\gamma$ of the $\operatorname{MEP}(2)$ class is that the joint density $(10), f\left(x_{0}, x_{1}, \ldots, x_{k}\right)$, must be non-negative. This constraint may be reformulated in terms of conditional densities. The $k$ th interarrival time with density

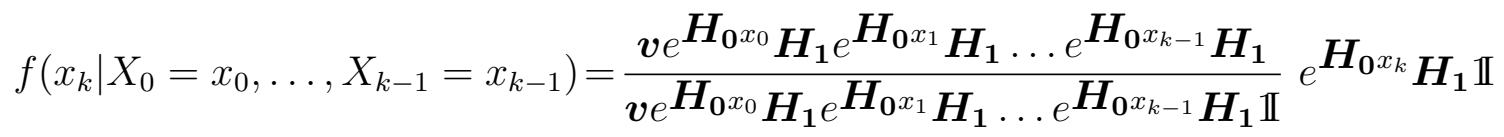

must be a valid $\operatorname{ME}(2)$ distribution $\forall k \geq 0$ and $\forall x_{1}, x_{2}, \ldots, x_{k} \geq 0$. Random variable $X_{k}$ has a valid $\mathrm{ME}(2)$ distribution, if its initial vector

$\boldsymbol{v}_{\boldsymbol{k}}\left(x_{0}, x_{1}, \ldots, x_{k-1}\right)=\frac{\boldsymbol{v} e^{\boldsymbol{H}_{\mathbf{0}} x_{0}} \boldsymbol{H}_{\mathbf{1}} e^{\boldsymbol{H}_{\mathbf{0}} x_{1}} \boldsymbol{H}_{\mathbf{1}} \ldots e^{\boldsymbol{H}_{\mathbf{0}} x_{k-1}} \boldsymbol{H}_{\mathbf{1}}}{\boldsymbol{v} e^{\boldsymbol{H}_{\mathbf{0}} x_{0}} \boldsymbol{H}_{\mathbf{1}} e^{\boldsymbol{H}_{\mathbf{0}} x_{1}} \boldsymbol{H}_{\mathbf{1}} \ldots e^{\boldsymbol{H}_{\mathbf{0}} x_{k-1}} \boldsymbol{H}_{\mathbf{1}} \mathbb{I}}$

is in the valid range defined by $\boldsymbol{H}_{\mathbf{0}}$. The valid range of representation (21) is

$0 \leq\left\{\boldsymbol{v}_{\boldsymbol{k}}\left(x_{0}, x_{1}, \ldots, x_{k-1}\right)\right\}_{1} \leq \frac{1}{1-a \alpha}=\frac{\lambda_{2}}{\lambda_{2}-\lambda_{1} a}$

according to representation (9) and Theorem 2. For second-order distributions, it is sufficient to check if the first element of vector $\boldsymbol{v}_{\boldsymbol{k}}$ falls into the valid range, since the property $\left\{\boldsymbol{v}_{\boldsymbol{k}}(\bullet)\right\}_{1}+\left\{\boldsymbol{v}_{\boldsymbol{k}}(\bullet)\right\}_{2}=1$ ensures the validity of the second element of $\boldsymbol{v}_{\boldsymbol{k}}(\bullet)$.

In fact, in Appendix B, we only discuss the validity of vector $\boldsymbol{v}_{\boldsymbol{k}}$ for two limiting cases, from which correlation bounds for parameter $\gamma$ are obtained. These bounds already constrain the permissible range of $\gamma$ to the one of the canonical $\operatorname{AMAP}(2)$ representation in Table 1 of Section 4.2 such that $M E P(2) \subset$ canonical $A M A P(2)$. Since the subset relation, $M E P(2) \supset M A P(2) \supset A M A P(2) \supset$ canonical $A M A P(2)$, is granted by the definition of the processes and the identity of the interarrival distributions is given by Theorem 2, the equivalence of Theorem 3 is then established. 
Theorem 3 The process sets $M E P(2), M A P(2)$ and $A M A P(2)$ are equivalent, i.e.,

$M E P(2) \equiv M A P(2) \equiv A M A P(2)$

The detailed proof is provided in Appendix B.

\section{Practical application of the canonical form for second-order processes}

In practical applications, two problems are often encountered: the inverse problem, where a process should be constructed from given traffic characteristics, and the transformation problem, where a given process representation should be transformed into another one, preferably to a canonical form (if available). The transformation may be motivated by obtaining a representation which expedites numerical procedures due to a sparser structure. In our context of second-order processes, queue analysis techniques or simulation procedures benefit from the triangular forms of matrices $\boldsymbol{D}_{\mathbf{0}}$ and $\boldsymbol{D}_{\mathbf{1}}$ (see canonical form of Definition 1) for arrival/service processes of a queue. Furthermore, some algorithms may even rely on a Markovian structure for a given process. Then, a given true MEP representation should be converted to a true MAP. In our case, this may be achieved by reducing the transformation problem to an inverse problem, i.e., traffic characteristics, like marginal moments and correlation coefficients, are computed from the $\operatorname{MEP}(2)$ and used to determine the parameters of the canonical form, which represents an $\operatorname{AMAP}(2)$.

In Section 4, we saw that four parameters are sufficient to characterize the stationary behavior of any second-order process, namely $\left(\lambda_{1}, \lambda_{2}, a, b\right)$ or alternatively $\left(\lambda_{1}, \alpha, p, \gamma\right)$. However, these parameters are not commonly observed, measured or computed in applications. In Section 6.1, we provide a mapping to construct a canonical $\operatorname{AMAP}(2)$ based on the first three moments of the interarrival times, $\mu_{1}, \mu_{2}, \mu_{3}$ (see (11)), and on the lag-1 correlation coefficient, as defined in (12) for $k=1$. Note that due to the equivalences proved in Section 5 any second-order process can be represented by the canonical form. Of course, a mapping procedure may start in principle from any four (sufficiently different) traffic characteristics and we discuss this issue in Section 6.2.

\subsection{Matching moments and correlation}

The matching of three moments and the lag- 1 correlation coefficient means to solve a system of four non-linear equations. Three equations are given by (11) for $n=1,2,3$ with matrix $\boldsymbol{H}_{\mathbf{0}}=\boldsymbol{D}_{\mathbf{0}}$ of the canonical form from Definition 1. An additional equation specifies (positive or negative) $\gamma$ according to equation (13) or (14), respectively. Parameter $\gamma$ itself is determined from the lag-1 correlation coefficient via (12) with $k=1$.

The symbolic solution of this system of equations is very cumbersome. Instead of solving it directly, we decompose the fitting procedure into two steps in analogy to [14]. Here, however, the fitting results in the $\operatorname{AMAP}(2)$ canonical form, which has one parameter less than the form in [14].

Step 1: obtain an $\operatorname{APH}(2)$ representation (8) based on the three moments. This step provides $\lambda_{1}, \lambda_{2}$ and $p$. At this point, the parameter set $\left(\lambda_{1}, \alpha, p, \gamma\right)$ is available, where $\alpha=\frac{\lambda_{1}}{\lambda_{2}}$. 
Step 2: transform the parameter set $\left(\lambda_{1}, \alpha, p, \gamma\right)$ into the equivalent parameter set $\left(\lambda_{1}, \lambda_{2}, a, b\right)$, in terms of which the canonical form is given. This step was already conducted in Section 4.1.

\section{The fitting procedure}

To make the formulas compact, we introduce the following moment expressions related to Hankel determinants (see also [13]):

$$
h_{1}=\mu_{1}, \quad h_{2}=\frac{\mu_{2}}{2 \mu_{1}^{2}}-1\left(=\frac{c_{v}^{2}-1}{2}\right), \quad h_{3}=\frac{\mu_{3}}{6 \mu_{1}^{3}}-\frac{\mu_{2}^{2}}{4 \mu_{1}^{4}} .
$$

By utilizing the results of [24], a canonical $\mathrm{APH}(2)$ can be constructed to describe the interarrival time based on the first three moments $\mu_{1}, \mu_{2}, \mu_{3}$ (or equivalently $h_{1}, h_{2}, h_{3}$ ). The resulting triple $\left(\lambda_{1}, \alpha, p\right)$ is the following:

$$
\begin{aligned}
\text { if } h_{2} \geq 0\left(c_{v}^{2} \geq 1\right): \quad \lambda_{i} & =\frac{h_{3}+h_{2}^{2}+h_{2} \mp \sqrt{\left(h_{3}+h_{2}^{2}+h_{2}\right)^{2}-4 h_{2} h_{3}}}{2 h_{1} h_{3}}, \quad i=1,2, \\
p & =\frac{-h_{3}-h_{2}^{2}+h_{2}+\sqrt{\left(h_{3}+h_{2}^{2}+h_{2}\right)^{2}-4 h_{2} h_{3}}}{h_{3}+h_{2}^{2}+h_{2}+\sqrt{\left(h_{3}+h_{2}^{2}+h_{2}\right)^{2}-4 h_{2} h_{3}}}, \quad \alpha=\frac{\lambda_{1}}{\lambda_{2}}, \\
\text { if } h_{2}<0\left(c_{v}^{2}<1\right): \quad \lambda_{i} & =\frac{h_{3}+h_{2}^{2}+h_{2} \pm \sqrt{\left(h_{3}+h_{2}^{2}+h_{2}\right)^{2}-4 h_{2} h_{3}}}{2 h_{1} h_{3}}, \quad i=1,2, \\
p & =\frac{h_{3}+h_{2}^{2}-h_{2}+\sqrt{\left(h_{3}+h_{2}^{2}+h_{2}\right)^{2}-4 h_{2} h_{3}}}{-h_{3}-h_{2}^{2}-h_{2}+\sqrt{\left(h_{3}+h_{2}^{2}+h_{2}\right)^{2}-4 h_{2} h_{3}}}, \quad \alpha=\frac{\lambda_{1}}{\lambda_{2}} .
\end{aligned}
$$

The case $h_{2}=0\left(c_{v}^{2}=1\right)$ is excluded in the following, because it leads to a degenerate representation stochastically equivalent to an exponential distribution.

Now parameters $a$ and $b$ are computed from (18), if $\gamma>0$, or from (20), if $\gamma<0$. For the sake of completeness, we repeat these formulas here:

$$
\begin{aligned}
\text { if } \gamma \geq 0: \quad a & =\frac{1}{2 \alpha}\left(1+\alpha \gamma-p(1-\gamma)-\sqrt{(1+\alpha \gamma-p(1-\gamma))^{2}-4 \alpha \gamma}\right) \\
b & =\frac{1}{2}\left(1+\alpha \gamma-p(1-\gamma)+\sqrt{(1+\alpha \gamma-p(1-\gamma))^{2}-4 \alpha \gamma}\right), \\
\text { if } \gamma \leq 0: \quad a & =\frac{-\gamma}{p(1-\gamma)-\alpha \gamma}, \quad b=p(1-\gamma)-\alpha \gamma .
\end{aligned}
$$

Note that we explicitly included the uncorrelated case $\gamma=0$ for both canonical forms, which lead to the same representation with $a=0, b=1-p$ and $a=0, b=p$, respectively.

Boundary conditions apply both to the correlation parameter $\gamma$ (as discussed in Appendix A) and to the moments. Without checking the boundary conditions for $h_{1}, h_{2}, h_{3}$ and $\gamma$ beforehand, the fitting procedure (24) and (25) may fail (e.g., due to negative discriminants). An indirect check of the validity of the input parameters consists of verifying whether the resulting canonical $\operatorname{AMAP}(2)$ is indeed a proper Markovian arrival process with well defined rate matrices.

However, available moment bounds and the correlation bounds derived in Appendix A enable the user to determine the validity of the input parameters in advance, as outlined in the next paragraphs. 
Table 2

Moment bounds for $h_{1}, h_{2}, h_{3}$

\begin{tabular}{lll}
\hline$h_{1}>0$ & hypoexponential & hyperexponential \\
\hline$h_{2}$ & $-\frac{1}{4} \leq h_{2}<0$ & $0<h_{2}$ \\
$h_{3}$ & $h_{2}\left(1-h_{2}-2 \sqrt{-h_{2}}\right) \leq h_{3} \leq-h_{2}{ }^{2}$ & $0<h_{3}$ \\
\hline
\end{tabular}

Table 3

Lower and upper $\gamma$-bounds in terms of $h_{2}$ and $h_{3}$

\begin{tabular}{llll}
\hline area & conditions & lower $\gamma$-bound & upper $\gamma$-bound \\
\hline I & $h_{2}<0$ & $-\left(\frac{h_{3}}{h_{2}}+h_{2}\right)$ & $-\frac{\left(\sqrt{\left.-h_{3}+h_{2}\right)^{2}}\right.}{h_{2}}$ \\
II & $h_{2}>0 \quad \& \quad \frac{h_{3}}{h_{2}}+h_{2}<1$ & $-\left(\frac{h_{3}}{h_{2}}+h_{2}\right)$ & 1 \\
III & $h_{2}>0 \& \quad 1 \leq \frac{h_{3}}{h_{2}}+h_{2}$ & $\frac{h_{3}+h_{2}^{2}-h_{2}-\sqrt{\left(h_{3}+h_{2}^{2}-h_{2}\right)^{2}+4 h_{2}^{3}}}{h_{3}+h_{2}^{2}-h_{2}+\sqrt{\left(h_{3}+h_{2}^{2}-h_{2}\right)^{2}+4 h_{2}^{3}}}$ & 1 \\
\hline
\end{tabular}

\section{Summary of moment and correlation bounds}

Initially, by means of Table 2 (taken from [14]), the validity of the first three moments $h_{1}, h_{2}, h_{3}$ can be ensured so that Step 1 of the fitting procedure, (24), is guaranteed to deliver permissible parameters, i.e., $\lambda_{1}>0$ and $\alpha, p \in[0,1]$.

Before Step 2 of the fitting procedure, the correlation bounds of Table 1 (in terms of $\alpha$ and $p$ ) allow one to check, if parameter $\gamma$ is within the permissible range. Any potentially unnecessary computation (like evaluating (24)) may be avoided, if one resorts to the correlation bounds in terms of the moment parameters $h_{2}, h_{3}$. Table 3 is simply a transformation of Table 1.

If $h_{1}, h_{2}, h_{3}$ and $\gamma$ fall within the respective bounds given by Tables 2 and 3 , the fitting procedure will always provide a valid canonical $\operatorname{AMAP}(2)$ representation, i.e., $\lambda_{2} \geq \lambda_{1}>0$ and $a, b \in[0,1]$.

\subsection{Disussion of the practical application}

We point out that especially fitting the correlation parameter $\gamma$ to a given correlation structure is not restricted to the lag-1 correlation coefficient. Instead of solving (12) for $\gamma$ with $k=1$, this equation uniquely determines a value for $\gamma$ for any (given) correlation coefficient at odd lag $k$. Furthermore, any pair of two adjacent correlation coefficients may be used to set $\gamma$ via $\gamma=\operatorname{Corr}\left(X_{0}, X_{k+1}\right) / \operatorname{Corr}\left(X_{0}, X_{k}\right)$. Such settings reflect the decay of the autocorrelation to some extent. Of course, $\gamma$-values determined in different ways may also be combined in a weighted sum in order to improve the fit to a given autocorrelation function as most appropriate for the considered application.

The fitting procedure of Section 6.1 matches the four parameters of the canonical form based on the first three moments and parameter $\gamma$ (e.g., related to the lag-1 correlation). If the inverse description for other four moment or correlation parameters is of interest, one can transform the given parameters to the first three marginal moments and the lag-1 correlation parameter according to [5]. Depending on the given moment and correlation parameters, this transformation can be unique (with explicit solution) or can have more 
than one (potentially only numerical) solutions out of which zero, one or more solutions may be feasible.

Generally, it is still an open research issue which traffic parameters characterize the performance-relevant properties of a traffic process best. Surely, the choice of parameters also depends on the specific application and performance measures to be computed. The fitting procedure of this section, however, shows that even second-order arrival processes already provide an astonishing flexibility in this mapping.

\section{Conclusions}

This paper proves the conjecture that the sets of second-order matrix-exponential processes $(\mathrm{MEP}(2) \mathrm{s})$ and second-order Markovian arrival processes (MAP $(2) \mathrm{s})$ are equivalent. As opposed to higher dimensions, the algebraic flexibility of $\operatorname{MEP}(2)$ s does not extend their modeling power over that of MAP(2)s. While this was known with respect to second-order distributions, the capability of these processes to capture correlations has not been fully characterized. Since we also showed that the sets of MAP(2)s and of acyclic $\mathrm{MAP}(2) \mathrm{s}$ are identical, we are now able to represent any of these processes $(\operatorname{MEP}(2), \operatorname{MAP}(2)$ or AMAP(2)) in an acyclic Markovian canonical form, which is introduced here for the first time. For this canonical $\operatorname{AMAP}(2)$ form, we explicitly computed the necessary and sufficient ranges of the single correlation parameter. Essentially, these bounds for $\operatorname{AMAP}(2)$ s were known before, but are now given in a substantially simpler form in terms of the parameters of the canonical form (and not in terms of the moments of the marginal distribution as in [14]). In fact, these simplified expressions only allowed us to prove the mentioned equivalence relations.

It is one thing to show properties of stochastic processes and another to actually construct these processes from moment/correlation information. We provided explicit analytical formulas to fit the first three moments of the marginal distribution and one correlation parameter to the parameters of the canonical $\operatorname{AMAP}(2)$ forms. This procedure also benefits from the more compact structure of the canonical forms (as compared to the one presented in [14]).

In conclusion, this paper reveals the relationships between second-order stochastic processes in matrix notation and in particular deepens the understanding of matrixexponential and Markovian traffic modeling. It remains to be seen inasmuch the results of this paper are helpful in the treatment of higher-dimensional and less-limited processes.

\section{REFERENCES}

1. A. T. Andersen and B. F. Nielsen. An application of superpositions of two-state Markovian sources to the modelling of self-similar behaviour. In Proc. 16th Annual Joint Conference of the IEEE Computer and Communications Societies, INFOCOM '97, volume 1, pages 196-204, Kobe, Japan, 1997.

2. S. Asmussen and M. Bladt. Point processes with finite-dimensional conditional probabilities. Stochastic Processes and their Applications, 82:127-142(16), July 1999.

3. S. Asmussen and C. A. O'Cinneide. Matrix-exponential distributions - distributions with a rational Laplace transform. In S. Kotz and C. Read, editors, Encyclopedia of Statistical Sciences, pages 435-440, New York, 1997. John Wiley \& Sons. 
4. A. Bobbio, A. Horváth, and M. Telek. Matching three moments with minimal acyclic phase-type distributions. Stochastic Models, 21(2-3):303-323, 2005.

5. L. Bodrog, A. Horváth, and M. Telek. Relation of moments and connected quantities in matrix-exponential and Markovian processes. Technical report, Technical University of Budapest, Dept. of Telecommunications, 2007. submitted for publication, http://webspn.hit.bme.hu/ telek/techrep/mom_relations.pdf.

6. D. R. Cox. A use of complex probabilities in the theory of stochastic processes. Proc. Cambridge Phil. Soc., 51:313-319, 1955.

7. S. Eum, R. Harris, and I. Atov. A matching model for MAP-2 using moments of the counting process. In Proc. International Network Optimization Conference, INOC 2007, Spa, Belgium, 2007.

8. M. Fackrell. Fitting with matrix-exponential distributions. Stochastic Models, 21:377400, 2005.

9. H.-W. Ferng and J.-F. Chang. Connection-wise end-to-end performance analysis of queueing networks with MMPP inputs. Performance Evaluation, 43:39-62, 2001.

10. Q. He and H. Zhang. A note on unicyclic representation of PH-distributions. Stochastic Models, 21:465-483, 2005.

11. Q. He and H. Zhang. Spectral polynomial algorithms for computing bi-diagonal representations for phase-type distributions and matrix-exponential distributions. Stochastic Models, 22(2):289-317, 2006.

12. H. Heffes and D. M. Lucantoni. A Markov-modulated characterization of packetized voice and data traffic and related statistical multiplexer performance. IEEE J. on Selected Areas in Commun., 4(6):856-868, 1986.

13. A. Heindl. Inverse characterization of hyperexponential MAP(2)s. In Proc. 11th Int. Conference on Analytical and Stochastic Modelling Techniques and Applications, pages 183-189, Magdeburg, Germany, 2004.

14. A. Heindl, G. Horváth, and K. Gross. Explicit inverse characterizations of acyclic MAPs of second-order. In A. Horváth and M. Telek, editors, Proc. 3rd European Performance Engineering Workshop, volume 4054 of LNCS, pages 108-122, Budapest, Hungary, 2006.

15. A. Heindl, K. Mitchell, and A. van de Liefvoort. Correlation bounds for second-order MAPs with application to queueing network decomposition. Performance Evaluation, 63:553-577, 2006.

16. G. Horváth, P. Buchholz, and M. Telek. A MAP fitting approach with independent approximation of the inter-arrival time distribution and the lag correlation. In Proc. 2nd Int. Conf. on Quantitative Evaluation of Systems, pages 124-133, Torino, Italy, 2005.

17. M. A. Johnson and M. R. Taaffe. Matching moments to phase distributions: Mixtures of Erlang distributions of common order. Commun. Statist.-Stochastic Models, 5(4):711-743, 1989.

18. S. H. Kang, Yong Han Kim, D. K. Sung, and B. D. Choi. An application of Markovian arrival process (MAP) to modeling superposed ATM cell streams. IEEE Transactions on Communications, 50(4):633-642, 2002.

19. S. H. Kang and D. K. Sung. Two-state MMPP modeling of ATM superposed traffic streams based on the characterization of correlated interarrival times. In Proc. Global 
Telecommunications Conference, GLOBECOM '95, volume 2, pages 1422-1426, Singapore, 1995.

20. L. Lipsky. Queueing Theory: A linear algebraic approach. MacMillan, New York, 1992.

21. D. Lucantoni, K. Meier-Hellstern, and M. Neuts. A single-server queue with server vacations and a class of non-renewal arrival processes. Advances in Applied Probability, 22:676-705, 1990.

22. K. Mitchell and A. van de Liefvoort. Approximation models of feed-forward G/G/1/N queueing networks with correlated arrivals. Performance Evaluation, 51:137-152, 2003.

23. M. Neuts. Matrix-Geometric Solutions in Stochastic Models. John Hopkins University Press, Baltimore, MD, USA, 1981.

24. M. Telek and A. Heindl. Matching moments for acyclic discrete and continuous phasetype distributions of second order. Int. Journal of Simulation, 3(3-4):47-57, 2003.

25. A. van de Liefvoort. The moment problem for continuous distributions. Technical Report WP-CM-1990-02, School of Computing and Engineering, University of Missouri - Kansas City, USA, 1990.

26. B. Wydrowski, C. H. Foh, B. Meini, and M. Zukerman. Analytical performance evaluation of a two class DiffServ link. In Proc. 8th Int. Conf. on Communication Systems, Singapore, November 2002.

\section{Appendix}

\section{A. Derivation of correlation bounds for canonical $\operatorname{AMAP}(2) \mathrm{s}$}

We first need to express $\gamma$ as a function of parameter $a$ (and for given $\alpha$ and $p$ ). In order to obtain the positive upper bounds for $\gamma$, we maximize this function with respect to $a$. Analogously, to obtain the negative lower bounds for $\gamma$, this function is minimized with respect to $a$. We consider these two cases in the following two subsections, respectively. At the beginning of each subsection, we first investigate if the a priori restriction that $\gamma \in[-1,1)$ is further constrained by the fact that parameter $b$ of the canonical form must be in $[0,1]$. Recall that a transformation from a permissible parameter set $\lambda_{1}, \alpha, p, \gamma$ to $\lambda_{1}, \lambda_{2}, a, b$ must not result in invalid parameters.

\section{A.1. Upper bounds for correlation parameter $\gamma$}

When computing the upper/positive correlation bounds $(\gamma>0)$, parameter $b$ can be expressed from equations (17) and (13) as:

$b=1-\frac{p}{1-\alpha a}(1-\gamma)$,

where $\frac{p}{1-\alpha a} \in[0,1]$, if $a$ is valid. From (26), it follows that any positive $\gamma$ may be chosen for $b$ to be in $[0,1]$, i.e., $b \in[0,1]$ does not impose an additional constraint on $\gamma$.

To find the upper bound, we express $b$ from (17), substitute it into (13), and obtain $\gamma$ as a function of $a$ :

$\gamma(a)=\frac{a(1-\alpha a-p)}{1-\alpha a-a p}$ 
The upper correlation bound is the maximum of this function with respect to a valid parameter $a$. This function has always two roots, one at $a=0$ and one at $a=\frac{1}{\alpha}(1-p)$.

The derivative of $\gamma(a)$ is the following:

$$
\frac{d}{d a} \gamma(a)=\frac{1-2 \alpha a-p}{1-\alpha a-a p}+\frac{a(1-\alpha a-p)(\alpha+p)}{(1-\alpha a-a p)^{2}}
$$

To obtain the maximum value, we look for $a^{(M A X)}$, for which $\left.\frac{d}{d a} \gamma(a)\right|_{a=a^{(M A X)}}=0$. There are two solutions:

$$
a_{i}^{(M A X)}=\frac{\alpha \pm \sqrt{p \alpha(\alpha+p-1)}}{\alpha^{2}+\alpha p}, \quad i=1,2 .
$$

For assessing the potential extrema, we need to consider the permissible range of parameter $a$, which was determined in Section 4.2 (after Lemma 1) and shown to depend on $c_{v}^{2}$. Therefore, we distinguish the two subcases $c_{v}^{2}<1$ and $c_{v}^{2}>1$.

Subcase $c_{v}^{2}<1$, where $a \in\left[0, \frac{1-p}{\alpha}\right]$

In this case, function $\gamma(a)$ has a maximum in the valid region, since it has roots at the borders of the valid region (at $a=0$ and at $a=\frac{1-p}{\alpha}$ ) and has a positive derivative at $a=0$. This maximum is tighter than $\gamma(a)=1$, since - if $a<1$ - it always holds that $\gamma(a)<1$ according to (27). Note that $a=1$ is not admitted for $\gamma>0$ (see first canonical form in Definition 1).

From the two solutions of $\frac{d}{d a} \gamma(a)=0$, only the one where the square root appears with negative sign is a real maximum, since the second derivative is negative only in this case. Thus, parameter $a$ that maximizes $\gamma(a)$ is:

$a^{(M A X)}=\frac{\alpha-\sqrt{p \alpha(\alpha+p-1)}}{\alpha^{2}+\alpha p}$.

Finally the upper limit of $\gamma$ is:

$\gamma^{(M A X)}=\gamma\left(a^{(M A X)}\right)=\frac{\alpha+p(\alpha+p-1)-2 \sqrt{p \alpha(-1+p+\alpha)}}{(p+\alpha)^{2}}$.

Subcase $c_{v}^{2}>1$, where $a \in[0,1]$

In this case, function $\gamma(a)$ is positive and increases monotonically in $\left(0, \frac{1}{\alpha+p}\right)$. From Lemma 1, it follows that if $c_{v}^{2}>1$ holds, $\frac{1}{\alpha+p}>1$. Therefore the maximum of $\gamma(a)$ is at the right-hand border of the valid region, i.e., at $a=1$ :

$\gamma^{(M A X)}=\left.\gamma(a)\right|_{a=1}=1$.

\section{A.2. Lower bounds for correlation parameter $\gamma$}

In order to check if $b \in[0,1]$ imposes an additional constraint on $\gamma<0$ (i.e., an additional lower/negative correlation bound), we use equations (19) and (14) to express parameter $b$ as

$b=(1-\gamma) \frac{p}{1-\alpha a}$. 
Obviously, this expression can be greater than 1 for $\gamma<0$ (as $\frac{p}{1-\alpha a} \in[0,1]$ ). This means that - for $b$ to be in $[0,1]$ - we have the following lower bound for $\gamma$ :

$\gamma \geq \frac{p+\alpha a-1}{p}=\tilde{\gamma}(a)$

In other words, the condition $b \in[0,1]$ mandates that $\tilde{\gamma}(a) \leq \gamma<0$.

To find the lowest permissible value of $\gamma$, we first express $\gamma$ as a function of $a$ via (14) and (19):

$\hat{\gamma}(a)=\frac{-a p}{1-a \alpha-a p}$.

We must minimize this function with respect to $a$ without violating $\gamma>\tilde{\gamma}(a)$ (see (30)). Let us first determine the range of $a$, where the lower bound $\tilde{\gamma}(a)$ supersedes $\hat{\gamma}(a)$, i.e., where $\hat{\gamma}(a) \leq \tilde{\gamma}(a)$ holds:

$\frac{-a p}{1-a \alpha-a p} \leq \frac{p+\alpha a-1}{p}$.

Solving this inequality, we find that $\tilde{\gamma}(a)$ is tighter, if

$a \geq \frac{1-p}{p+\alpha}$.

Thus the possible cases are:

- If $a<\frac{1-p}{p+\alpha}$, the lower bound is provided by the minimum of $\hat{\gamma}(a)$ in the range $a=\left(0, \frac{1-p}{p+\alpha}\right)$. Since $\hat{\gamma}(a)$ decreases monotonically, the minimum is reached for the largest possible value of $a$.

- If $a \geq \frac{1-p}{p+\alpha}$, the lower bound is provided by $\tilde{\gamma}(a)$. But $\tilde{\gamma}(a)$ increases monotonically with $a$. Thus the smallest value of the lower bound is reached for the smallest value of $a: a=\frac{1-p}{p+\alpha}$.

Based on this discussion, the optimal parameter $a$ is $a^{(M I N)}=\frac{1-p}{p+\alpha}$, if it is permissible.

The permissible ranges of parameter $a$ depend on $c_{v}^{2}$ (see Section 4.2, after Lemma 1) so that we again treat the two subcases $c_{v}^{2}<1$ and $c_{v}^{2}>1$ separately:

Subcase $c_{v}^{2}<1$, where $a \in\left[0, \frac{1-p}{\alpha}\right]$

Since $\frac{1-p}{p+\alpha}<\frac{1-p}{\alpha}$, the above choice of parameter $a$ is optimal and permissible:

$a^{(M I N)}=\frac{1-p}{p+\alpha}$.

At this point, both $\tilde{\gamma}(a)$ and $\hat{\gamma}(a)$ are equal and provide the lower limit for $\gamma$ :

$\gamma^{(M I N)}=-\frac{1-p}{p+\alpha}$. 
Subcase $c_{v}^{2}>1$, where $a \in[0,1]$

In this case, $\frac{1-p}{p+\alpha}<1$ does not necessarily hold.

- If $\frac{1-p}{p+\alpha}<1$, then we have the same optimal and permissible parameter $a$ and the same lower bound as in the case $c_{v}^{2}<1$. Therefore,

$\gamma^{(M I N)}=-\frac{1-p}{p+\alpha}$.

- If $\frac{1-p}{p+\alpha} \geq 1$, then the lower bound is determined by $\hat{\gamma}(a)$. Since $\hat{\gamma}(a)$ decreases monotonically, the optimal parameter $a$ is located at the upper border of the valid range, $a^{(M I N)}=1$, and

$\gamma^{(M I N)}=\frac{p}{p+\alpha-1}$.

\section{B. Proof of Theorem 3}

The proof of Theorem 3 is based on the study of the permissible range of parameter $\gamma$ for $\mathrm{MEP}(2) \mathrm{s}$. It turns out that this permissible range for $\mathrm{MEP}(2) \mathrm{s}$ is identical with the one of $\operatorname{AMAP}(2) \mathrm{s}$.

In fact, as outlined before Theorem 3 in Section 5, it suffices to consider two limiting cases, namely that the interarrival periods are very short or very long. We treat these two cases in Sections B.1 and B.2, respectively. Essentially, necessary conditions for the initial vector $(22)$ of the $k$ th interarrival time distribution are exploited to derive correlation bounds. We indicate the two cases by the notation

$$
\begin{aligned}
\boldsymbol{v}_{\boldsymbol{k}}(\mathbf{0}) & =\lim _{x_{0} \backslash 0, x_{1} \backslash 0, \ldots, x_{k-1} \backslash 0} \boldsymbol{v}_{\boldsymbol{k}}\left(x_{0}, x_{1}, \ldots, x_{k-1}\right) \\
\boldsymbol{v}_{\boldsymbol{k}}(\infty) & =\lim _{x_{0} / \infty, x_{1} \nearrow, \ldots, x_{k-1} \nearrow \infty} \boldsymbol{v}_{\boldsymbol{k}}\left(x_{0}, x_{1}, \ldots, x_{k-1}\right) .
\end{aligned}
$$

\section{B.1. Necessary conditions based on $\boldsymbol{v}_{\boldsymbol{k}}(\mathbf{0})$}

To analyze the sequence of initial vectors $\boldsymbol{v}_{\boldsymbol{k}}(\mathbf{0})$, where $k \geq 1$, we define the function $\hat{u}(x, t)$ that expresses the first element of the initial vector for $X_{k}$ $\left(\left\{\boldsymbol{v}_{\boldsymbol{k}}\left(x_{0}, x_{1}, \ldots, x_{k-2}, t\right)\right\}_{1}\right)$ as a function of the first element of the initial vector for $X_{k-1}$ $\left(\left\{\boldsymbol{v}_{\boldsymbol{k}-\mathbf{1}}\left(x_{0}, x_{1}, \ldots, x_{k-2}\right)\right\}_{1}=x\right)$ assuming $X_{k-1}=t$ :

$\hat{u}(x, t)=\left\{\frac{[x, 1-x] e^{\boldsymbol{H}_{\mathbf{0}} t} \boldsymbol{H}_{\mathbf{1}}}{[x, 1-x] e^{\boldsymbol{H}_{\mathbf{0}^{t}} \boldsymbol{H}_{\mathbf{1}} \mathbb{I}}}\right\}_{1}$.

In case of representation $(21)$

$\hat{u}(x, t)=\frac{e^{-\lambda_{1} t} x \alpha(a \alpha-1)(p(\gamma-1)+\gamma(\alpha-1))-e^{-\lambda_{1} t / \alpha} p(1-\alpha+x(a \alpha-1))(\gamma-1)}{(a \alpha-1)\left(e^{-\lambda_{1} t} x \alpha(a \alpha-1)+e^{-\lambda_{1} t / \alpha}(x+\alpha-a x \alpha-1)\right)}$.

Substituting $t=0$ results in

$$
u(x)=\hat{u}(x, 0)=\frac{p(1+x(a \alpha-1))(\gamma-1)+x \alpha \gamma(a \alpha-1)}{(a \alpha-1)(1+x(a \alpha-1))} .
$$


Let $w_{k}$ be the first element of vector $\boldsymbol{v}_{\boldsymbol{k}}(\mathbf{0})$ and $\underline{w}=0$ and $\bar{w}=\frac{1}{1-a \alpha}$ the lower and upper bound, respectively, of valid $\mathrm{ME}(2)$ distributions as defined in (23). Element $w_{k}$ can be obtained successively from $u(x)$ via

$w_{0}=\frac{p}{1-a \alpha}, w_{1}=u\left(w_{0}\right), w_{2}=u\left(w_{1}\right), w_{3}=u\left(w_{2}\right), \ldots$

To ensure that $\underline{w} \leq w_{k} \leq \bar{w} \forall k \geq 0, u(x)$ has to fulfill the following necessary constraints:

$C 1: x=u(x)$ has a solution in range $[\underline{w}, \bar{w}]$ denoted by $x^{*}$, and

C2: $-1 \leq u^{\prime}\left(x^{*}\right) \leq 1$.

C3: If $x=u(x)$ has another solution in range $[\underline{w}, \bar{w}]$ denoted by $x^{* *}$ and $x^{*}<x^{* *}$, then $\underline{w}<w_{0}=\frac{p}{1-a \alpha}<x^{* *}$.

There are further obvious necessary constraints, but we use only these three in this subsection. The intuitive explanation of these constraints is as follows: $u(x)$ is a hyperbola. If $u(x)=x$ has two solutions in range $(\underline{w}, \bar{w})$, one satisfies and one violates $C 2$. The vertical axis of the hyperbola is at $x=\frac{p}{1-a \alpha}$. If $\gamma>0(\gamma<0)$ then $u(x)$ is increasing (decreasing) on both sides of this vertical axis (Figure 4).
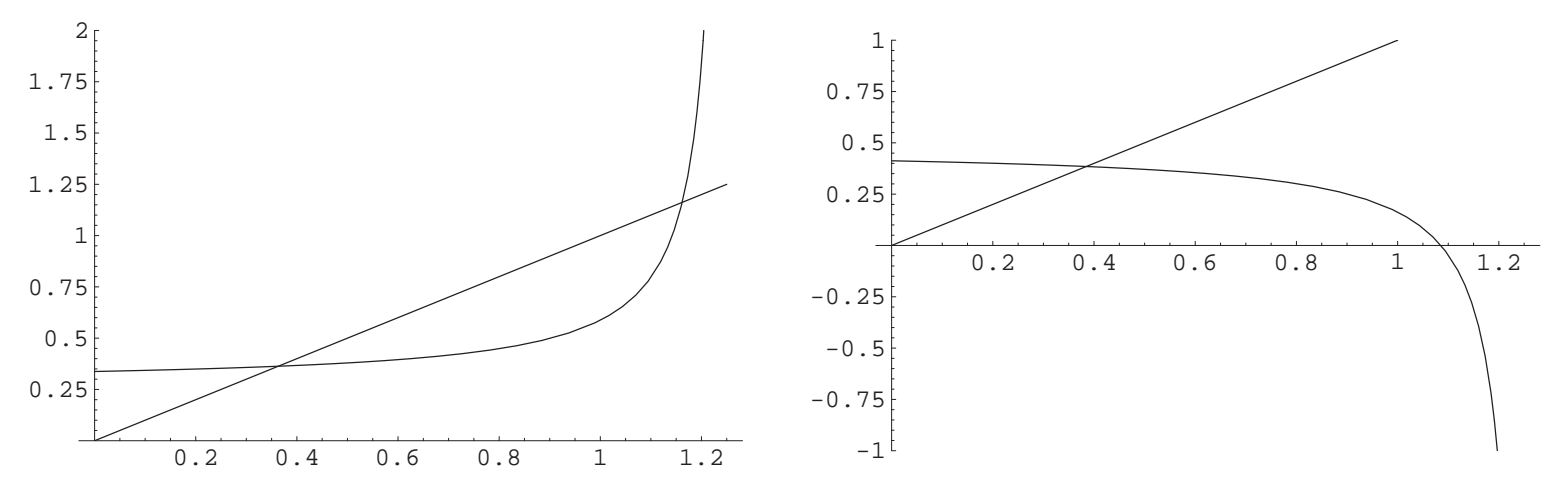

Figure 4. Exemplary behavior of equation $u(x)=x$ with $\gamma>0$ (left) and $\gamma<0$ (right) for $a=0.4, \gamma= \pm 0.1, p=0.3, \alpha=0.5$

If $C 1$ is violated such that $x>u(x)(x<u(x))$ in the range $\underline{w} \leq x \leq \bar{w}$, then the series $w_{k}$ eventually decreases below $\underline{w}$ (increases above $\bar{w}$ ). If $C 2$ is violated, then starting from a point different from $x^{*}$, i.e., $w_{0} \neq x^{*}$, the series $w_{k}$ diverges away from $x^{*}$, either out of the interval $[\underline{w}, \bar{w}]$ or to another point $x^{\bullet}$, which satisfies $C 1$ and C2. This case of another solution, i.e., when $x=u(x)$ has two solutions in the range $(\underline{w}, \bar{w})$, is considered in $C 3$. If we have $\underline{w}<x^{*}<x^{* *}<\bar{w}$, then - starting from the interval $\left(\underline{w}, x^{* *}\right)$ (i.e., $w_{0} \in\left(\underline{w}, x^{* *}\right)$ ) - $w_{k}$ converges to $x^{*}$, but - starting from the interval $\left(x^{* *}, \bar{w}\right)$ (i.e., $\left.w_{0} \in\left(x^{* *}, \bar{w}\right)\right)-w_{k}$ increases above $\bar{w}$. 
Constraint C1: $x=u(x)$ is a quadratic equation in $x$, whose solutions are

$$
x_{i}^{*}=\frac{p(1-\gamma)+(1-\alpha \gamma) \mp \sqrt{p^{2}(1-\gamma)^{2}+(1-\alpha \gamma)^{2}-2 p(1-\gamma)(\alpha \gamma+1)}}{2(1-a \alpha)}, \quad i=1,2 .
$$

It has a real solution, if

$p^{2}(1-\gamma)^{2}+(1-\alpha \gamma)^{2}-2 p(1-\gamma)(\alpha \gamma+1) \geq 0$.

When $\gamma<0$, inequality (36) holds. When $\gamma>0$, we have two cases. If $c_{v}^{2}>1$ (i.e., $p+\alpha-1<0$ ), then (36) is satisfied. If $c_{v}^{2}<1$ (i.e., $p+\alpha-1>0$ ), then (36) represents a necessary constraint for $\gamma$ as a function of $p$ and $\alpha$. Correlation parameter $\gamma$ should satisfy

$\gamma \leq \frac{p(p-1)+p \alpha+\alpha-2 \sqrt{p \alpha(p+\alpha-1)}}{(p+\alpha)^{2}}$ or $\gamma \geq \frac{p(p-1)+p \alpha+\alpha+2 \sqrt{p \alpha(p+\alpha-1)}}{(p+\alpha)^{2}}$

According to constraint $C 3$ and the following Lemma, the second part of (37) is not valid, which leaves the necessary condition

$\gamma \leq \frac{p(p-1)+p \alpha+\alpha-2 \sqrt{p \alpha(p+\alpha-1)}}{(p+\alpha)^{2}}$

when $\gamma>0$ and $p+\alpha-1>0$. Note the identity of the bound expression with (28).

Lemma 2 When $p+\alpha-1>0$ and

$\gamma \geq \frac{p(p-1)+p \alpha+\alpha+2 \sqrt{p \alpha(p+\alpha-1)}}{(p+\alpha)^{2}}$

then $x_{2}^{*}<\frac{p}{1-a \alpha}$.

Proof: Based on the definition of $x_{2}^{*}$ in (35), we need to show that

$$
2 p-(p(1-\gamma)+(1-\alpha \gamma))>\sqrt{p^{2}(1-\gamma)^{2}+(1-\alpha \gamma)^{2}-2 p(1-\gamma)(\alpha \gamma+1)} .
$$

We show this in two steps. First we show that the lhs of (40) is positive, and then we show that the square of the lhs of (40) is greater than the square of the rhs. Multiplying the lhs with $(p+\alpha)$ yields

$$
\begin{aligned}
& {[2 p-(p(1-\gamma)+(1-\alpha \gamma))](p+\alpha)=(p+(p+\alpha) \gamma-1)(p+\alpha) \geq} \\
& p(p+\alpha)+p(p-1)+p \alpha+\alpha+2 \sqrt{p \alpha(p+\alpha-1)}-(p+\alpha)= \\
& 2 p(p+\alpha-1)+2 \sqrt{p \alpha(p+\alpha-1)}>0,
\end{aligned}
$$


where we substituted the lower bound of $\gamma(39)$ in the second step and applied the condition $p+\alpha-1>0$ in the last one.

The square of the lhs of (40) minus the square of its rhs is

$$
4 p^{2}-4 p(p(1-\gamma)+(1-\alpha \gamma))+4 p(1-\gamma)=4 p \gamma(p+\alpha-1)>0
$$

which proves the lemma.

Now, we consider the effect of constraint C2, when $\gamma<0$. In this case, equation $u(x)=x$ has exactly one solution in $[\underline{w}, \bar{w}]$ and $u(x)$ is decreasing, i.e., $u^{\prime}(x)<0$ (see Figure 4). Substituting $x_{1}^{*}$ from (35) into $u^{\prime}(x)$, we obtain that $u^{\prime}\left(x^{*}\right) \geq-1$ if

$\gamma \geq-\frac{1-p}{p+\alpha}$

We can summarize the results of this subsection by means of Figure 3 in Section 4.2, which depicts the $(p, \alpha)$-plane. In area I, we have $c_{v}^{2}<1(p+\alpha>1)$ and (38) upper bounds $\gamma$. In areas II and III, we have $c_{v}^{2}>1(p+\alpha<1)$ and the constraints do not upper bound $\gamma$ (i.e., $\gamma<1$ ). Expression (43) lower bounds $\gamma$, but this lower bound is meaningless (less than -1 ) in area III and effective in areas I and II only.

\section{B.2. Necessary condition based on $\boldsymbol{v}_{\boldsymbol{k}}(\infty)$}

Substituting $t=\infty$ into (34) for $\hat{u}(x, t)$ results in

$\tilde{u}(x)=\hat{u}(x, \infty)=\frac{p(\gamma-1)+\gamma(\alpha-1)}{a \alpha-1}$.

Note that $\tilde{u}(x)$ is actually independent of $x$. Due to this independence, it is sufficient to check, if $w_{1}=\left\{\boldsymbol{v}_{1}(\infty)\right\}_{1}=\tilde{u}(x)$ is in the interval $[\underline{w}, \bar{w}]$. From $\underline{w}=0 \leq \tilde{u}(x)$ we obtain

$\gamma \geq \frac{p}{p+\alpha-1}$

Expression (44) provides another lower bound of $\gamma$ comparable to (33). This lower bound is meaningless (less than -1) in areas I and II of Figure 3 and effective in area III.

When we collect all the necessary constraints in this section and compare them with the correlation bounds for canonical $\operatorname{AMAP}(2) \mathrm{s}$ in Section 4.2, it is easily seen that $\gamma$ for $\operatorname{MEP}(2) \mathrm{s}$ is at least as restricted as the correlation parameter for canonical $\operatorname{AMAP}(2) \mathrm{s}$. Since the tuple $\left(\lambda_{1}, \alpha, p, \gamma\right)$ completely defines a second-order arrival process, this section proves that $M E P(2) \subset$ canonical $A M A P(2)$. Therefore, together with Section 4 , it holds that $M E P(2) \equiv M A P(2) \equiv A M A P(2)$. 